\title{
Well-posedness of the linear heat equation with a second order memory term
}

\author{
Constantin Niţă ${ }^{1, *}$ and Laurenţiu Emanuel Temereancă ${ }^{2, * *}$ \\ ${ }^{1}$ Department of Mathematics, University of Craiova, 200585, Romania \\ ${ }^{2}$ Department of Applied Mathematics, University of Craiova, 200585, Romania
}

\begin{abstract}
In this article we prove that the heat equation with a memory term on the one-dimensional torus has a unique solution and we study the smoothness properties of this solution. These properties are related with some smoothness assumptions imposed to the initial data of the problem and to the source term.
\end{abstract}

Keywords: heat equation, memory term

\section{Introduction and preliminaries}

Let $\mathbb{T}:=\mathbb{R} / 2 \pi \mathbb{Z}$ be the one-dimensional torus, let $Q:=(0, T) \times \mathbb{T}$ and $M>0$. In this article we study the existence and uniqueness of the solution of the following heat equation involving a memory term:

$$
\begin{cases}u_{t}(t, x)-u_{x x}(t, x)+M \int_{0}^{t} u_{x x}(s, x) \mathrm{ds}=f(t, x) & (t, x) \in Q \\ u(0, x)=u^{0}(x) & x \in \mathbb{T},\end{cases}
$$

where the source term $f \in L^{2}\left((0, T), L^{2}(\mathbb{T})\right)$.

In many problems arising in mathematical physics such as flow of fluid through fissured rocks, diffusion process of gas in a transparent tube, heat conduction in materials, and viscoelasticity, one may encounter memory effects that are relevant from a physical point of view and can be modeled by nonlocal terms. For instance, in [3], a modified Fourier's law which is independent of the present value of the temperature gradient, but depends on its history, is introduced to correct the unphysical property of instantaneous propagation for the heat equation. By following [1,5] (see, also, [2]) has been proposed a linearized theory for the heat transfer in isotropic media in which the heat flux depends both on the present value of the temperature gradient and its history being given by

$$
\vec{q}(t, x)=-k(0) \nabla \vartheta(t, x)-\int_{0}^{\infty} k^{\prime}(s) \nabla \vartheta(s, x) \mathrm{ds},
$$

where $\mathrm{k}(\mathrm{s})$ is the heat conduction relaxation function and $\vartheta$ represents the temperature. A particular and simplified case of this modified Fourier's law is used for the equation with memory term (1).

\footnotetext{
*e-mail: nita_gyk@yahoo.com

**e-mail: temereanca_laurentiu@yahoo.com
} 
Let us introduce the following space with its corresponding norm:

$$
L^{2}(\mathbb{T}):=\left\{f:\left.\mathbb{T} \rightarrow \mathbb{C}\left|\int_{-\pi}^{\pi}\right| f(x)\right|^{2} \mathrm{dx}<\infty\right\}, \quad\|f\|_{L^{2}(\mathbb{T})}:=\left(\frac{1}{2 \pi} \int_{-\pi}^{\pi}|f(x)|^{2} \mathrm{dx}\right)^{\frac{1}{2}} .
$$

We observe that (1) may be written as the following system

$$
\begin{cases}u_{t}(t, x)-u_{x x}(t, x)+M z(t, x)=f(t, x), & (t, x) \in Q \\ z_{t}(t, x)-u_{x x}(t, x)=0, & (t, x) \in Q \\ u(0, x)=u^{0}(x), & x \in \mathbb{T} \\ z(0, x)=z^{0}(x), & x \in \mathbb{T},\end{cases}
$$

which is equivalent to

$$
\left(\begin{array}{l}
u \\
z
\end{array}\right)_{t}+\mathcal{A}\left(\begin{array}{c}
u \\
z
\end{array}\right)=\left(\begin{array}{l}
f \\
0
\end{array}\right), \quad\left(\begin{array}{l}
u \\
z
\end{array}\right)(0)=\left(\begin{array}{c}
u^{0} \\
z^{0}
\end{array}\right),
$$

where $f \in L^{2}\left((0, T) ; L^{2}(\mathbb{T})\right)$ and the operator $\mathcal{A}$ is given by

$$
\mathcal{A}=\left(\begin{array}{cc}
-\partial_{x x}^{2} & M \mathrm{I}_{d} \\
-\partial_{x x}^{2} & 0
\end{array}\right)
$$

$\mathrm{I}_{d}$ being the identity operator. The domain of the operator $\mathcal{A}$ is given by $D(\mathcal{A})=H^{2}(\mathbb{T}) \times$ $L^{2}(\mathbb{T})$ and it is characterized by the following property

$$
\left(\begin{array}{c}
u^{0} \\
z^{0}
\end{array}\right) \in D(\mathcal{A}) \Leftrightarrow \mathcal{A}\left(\begin{array}{c}
u^{0} \\
z^{0}
\end{array}\right) \in\left(L^{2}(\mathbb{T})\right)^{2} .
$$

Notice that in (3) the function $z$ denotes $\int_{0}^{t} u_{x x}(s, x)$ ds and, consequently, $z(0, \cdot)=0$. However, we can solve (3) for arbitrary value $z(0, \cdot)=z^{0}$.

\section{Spectral analysis}

In this section we present the spectral analysis of the operator $\mathcal{A}$. We have the following result.

Lemma 2.1 The eigenvalues of the operator $\mathcal{A}$ are given by the family

$$
\left(\mu_{n}^{ \pm}\right)_{n \in \mathbb{N}^{*}} \cup\left\{\mu_{0}\right\}
$$

where $\mu_{0}=0$ and

$$
\mu_{n}^{ \pm}=\frac{1}{2}\left(n^{2} \pm n \sqrt{n^{2}+4 M}\right) \quad\left(n \in \mathbb{N}^{*}\right) .
$$

Moreover, each of the eigenvalues $\mu_{n}^{ \pm}, n \in \mathbb{N}^{*}$, is double and has associated two eigenvectors

$$
\Phi_{n}^{ \pm}=\left(\begin{array}{c}
1 \\
\frac{n^{2}}{\mu_{n}^{ \pm}}
\end{array}\right) e^{i n x}, \quad \Phi_{-n}^{ \pm}=\left(\begin{array}{c}
1 \\
\frac{n^{2}}{\mu_{n}^{ \pm}}
\end{array}\right) e^{-i n x} \quad\left(n \in \mathbb{N}^{*}\right) .
$$

The eigenvalue $\mu_{0}$ has associated one eigenvector $\Phi_{0}^{+}=\left(\begin{array}{l}1 \\ 0\end{array}\right)$ and a generalized eigenvector $\Phi_{0}^{-}=\left(\begin{array}{c}1 \\ \frac{1}{M}\end{array}\right)$ verifying $\mathcal{A} \Phi_{0}^{-}=\Phi_{0}^{+}$. 
Proof. Let $\mu \in \mathbb{C}$ such that $\mathcal{A}\left(\begin{array}{l}u \\ z\end{array}\right)=\mu\left(\begin{array}{l}u \\ z\end{array}\right)$. We obtain that

$$
\left\{\begin{array}{l}
-u_{x x}+M z=\mu u \\
-u_{x x}=\mu z
\end{array}\right.
$$

If we are looking for $u$ and $z$ in the form

$$
u=\sum_{n \in \mathbb{Z}} a_{n} e^{i n x}, \quad z=\sum_{n \in \mathbb{Z}} b_{n} e^{i n x},
$$

from (9) it follows that

$$
\left\{\begin{array}{l}
\left(n^{2}-\mu\right) a_{n}+M b_{n}=0 \quad(n \neq 0), \\
n^{2} a_{n}-\mu b_{n}=0 \quad(n \neq 0), \\
\mu b_{0}=0 \\
M b_{0}=\mu a_{0} .
\end{array}\right.
$$

The above system has a non-zero solution $\left(a_{n}, b_{n}\right)$ if $\mu$ verifies the following equation

$$
\mu^{2}-n^{2} \mu-n^{2} M=0 \quad(n \neq 0) .
$$

Solving (10) we obtain that $\mu$ are given by (7), for each $n \in \mathbb{N}^{*}$. Since we have $z=\frac{-u_{x x}}{\mu}$ we deduce the form (8) for the eigenvectors $\Phi_{n}^{ \pm}$and $\Phi_{-n}^{ \pm}$, for each $n \in \mathbb{N}^{*}$.

If $b_{0} \neq 0$ we obtain $\mu=M=0$. If $b_{0}=0$ we obtain $\mu=0$ is a double eigenvalue and the corresponding eigenvector and generalized eigenvector are given by $\Phi_{0}^{+}$and $\Phi_{0}^{-}$respectively, where $\Phi_{0}^{-}$is obtained by solving $\mathcal{A} \Phi_{0}^{-}=\Phi_{0}^{+}$. The proof is complete.

The following remark gives some useful properties of the eigenvalues given by (7).

Remark 2.1 We remark that, the eigenvalues $\left(\mu_{n}^{ \pm}\right)_{n \in \mathbb{N}^{*}}$ given by (7) can be written in the following form

$$
\mu_{n}^{+}=n^{2}+r_{n}, \quad \mu_{n}^{-}=-r_{n} \quad\left(n \in \mathbb{N}^{*}\right),
$$

where

$$
r_{n}:=\frac{2 M}{1+\sqrt{1+\frac{4 M}{n^{2}}}} \quad\left(n \in \mathbb{N}^{*}\right)
$$

Moreover, the following estimates hold

$$
\frac{n^{2}}{2} \leq\left|\mu_{n}^{+}\right| \leq \frac{(1+\sqrt{1+4 M}) n^{2}}{2}, \quad \frac{2 M}{1+\sqrt{1+4 M}} \leq\left|\mu_{n}^{-}\right| \leq M \quad\left(n \in \mathbb{N}^{*}\right) .
$$

Also, notice that

$$
\mu_{n}^{+}-\mu_{n}^{-}=2 r_{n}+n^{2}>\frac{4 M}{1+\sqrt{1+4 M}} .
$$

Theorem 2.1 For any $n \in \mathbb{N}^{*}$, let $\gamma_{n}^{ \pm}:=\left(\left(\frac{n^{2}}{\mu_{n}^{ \pm}}\right)^{2}+1\right)^{-\frac{1}{2}}$. Then $\left(\gamma_{|n|}^{ \pm} \Phi_{n}^{ \pm}\right)_{n \in \mathbb{Z}^{*}} \cup\left\{\Phi_{0}^{+}, \frac{M}{\sqrt{M^{2}+1}} \Phi_{0}^{-}\right\}$is a Riesz basis in the spaces $\left(L^{2}(\mathbb{T})\right)^{2}$.

Proof. Firstly, we show that $\left(\gamma_{|n|}^{ \pm} \Phi_{n}^{ \pm}\right)_{n \in \mathbb{Z}^{*}} \cup\left\{\Phi_{0}^{+}, \frac{M}{\sqrt{M^{2}+1}} \Phi_{0}^{-}\right\}$is complete in $\left(L^{2}(\mathbb{T})\right)^{2}$. We prove that, given an arbitrary element in $\left(L^{2}(\mathbb{T})\right)^{2}$,

$$
\left(\begin{array}{c}
v \\
w
\end{array}\right)=\sum_{n \in \mathbb{Z}}\left(\begin{array}{c}
\beta_{n}^{+} \\
\beta_{n}^{-}
\end{array}\right) e^{i n x}
$$


there exists a unique sequence $\left(a_{n}^{ \pm}\right)_{n \in \mathbb{Z}}$ such that

$$
\left(\begin{array}{c}
v \\
w
\end{array}\right)=\sum_{n \in \mathbb{Z}^{*}} a_{n}^{ \pm} \gamma_{|n|}^{ \pm} \Phi_{n}^{ \pm}+a_{0}^{+} \Phi_{0}^{+}+a_{0}^{-} \Phi_{0}^{-}
$$

From the form (8) of $\Phi_{n}^{ \pm}$, the form of $\Phi_{0}^{+}, \Phi_{0}^{-}$given by Lemma 2.1 and by relations (14) and (15) we obtain the following system

$$
\left\{\begin{array}{l}
a_{n}^{+} \gamma_{|n|}^{+}+a_{n}^{-} \gamma_{|n|}^{-}=\beta_{n}^{+}, \quad n \neq 0 \\
a_{n}^{+} \gamma_{|n|}^{+} \frac{n^{2}}{\mu_{|n|}^{+}}+a_{n}^{-} \gamma_{|n|}^{-} \frac{n^{2}}{\mu_{|n|}^{-}}=\beta_{n}^{-}, \quad n \neq 0 \\
a_{0}^{+}+\frac{M}{\sqrt{M^{2}+1}} a_{0}^{-}=\beta_{0}^{+}, \\
\frac{1}{\sqrt{M^{2}+1}} a_{0}^{-}=\beta_{0}^{-}
\end{array}\right.
$$

By taking into account (13) it results that the above system has a unique solution $a_{n}^{ \pm}$given by

$$
\left\{\begin{array}{l}
a_{n}^{ \pm}=\frac{\left(\beta_{n}^{+} n^{2}-\mu_{\mid n}^{\mp} \beta_{n}^{-}\right) \mu_{|n|}^{ \pm}}{\gamma_{|n|}^{ \pm} n^{2}\left(\mu_{|n|}^{ \pm}-\mu_{|n|}^{\mp}\right)}, \quad n \neq 0 \\
a_{0}^{-}=\sqrt{M^{2}+1} \beta_{0}^{-} \\
a_{0}^{+}=\beta_{0}^{+}-M \beta_{0}^{-}
\end{array}\right.
$$

and thus $\left(\gamma_{|n|}^{ \pm} \Phi_{n}^{ \pm}\right)_{n \in \mathbb{Z}^{*}} \cup\left\{\Phi_{0}^{+}, \frac{M}{\sqrt{M^{2}+1}} \Phi_{0}^{-}\right\}$is complete in $\left(L^{2}(\mathbb{T})\right)^{2}$. The proof is finished if we shows that $\left(\gamma_{|n|}^{ \pm} \Phi_{n}^{ \pm}\right)_{n \in \mathbb{Z}^{*}} \cup\left\{\Phi_{0}^{+}, \frac{M}{\sqrt{M^{2}+1}} \Phi_{0}^{-}\right\}$is a Riesz sequence in $\left(L^{2}(\mathbb{T})\right)^{2}$, that is, there exist two positive constants $K_{1}$ and $K_{2}$ such that

$$
K_{1}\left(\sum_{n \in \mathbb{Z}}\left|a_{n}^{ \pm}\right|^{2}\right) \leq\left\|\sum_{n \in \mathbb{Z}^{*}} a_{n}^{ \pm} \gamma_{|n|}^{ \pm} \Phi_{n}^{ \pm}+a_{0}^{+} \Phi_{0}^{+}+a_{0}^{-} \frac{M}{\sqrt{M^{2}+1}} \Phi_{0}^{-}\right\|_{\left(L^{2}(\mathbb{T})\right)^{2}}^{2} \leq K_{2}\left(\sum_{n \in \mathbb{Z}}\left|a_{n}^{ \pm}\right|^{2}\right),
$$

for all sequences of scalars $\left(a_{n}^{ \pm}\right)_{n \in \mathbb{Z}}$ in the space $\ell^{2}$. We have that

$$
\begin{aligned}
& \left\|\sum_{n \in \mathbb{Z}^{*}} a_{n}^{ \pm} \gamma_{|n|}^{ \pm} \Phi_{n}^{ \pm}+a_{0}^{+} \Phi_{0}^{+}+a_{0}^{-} \frac{M}{\sqrt{M^{2}+1}} \Phi_{0}^{-}\right\|_{\left(L^{2}(\mathbb{T})\right)^{2}}^{2} \\
& =\left\|\sum_{n \in \mathbb{Z}^{*}}\left(a_{n}^{+} \gamma_{|n|}^{+}+a_{n}^{-} \gamma_{|n|}^{-}\right) e^{i n x}+a_{0}^{+}+a_{0}^{-} \frac{M}{\sqrt{M^{2}+1}}\right\|_{L^{2}(\mathbb{T})}^{2} \\
& +\left\|\sum_{n \in \mathbb{Z}^{*}}\left(a_{n}^{+} \gamma_{|n|}^{+} \frac{n^{2}}{\mu_{|n|}^{+}}+a_{n}^{-} \gamma_{|n|}^{-} \frac{n^{2}}{\mu_{|n|}^{-}}\right) e^{i n x}+a_{0}^{-} \frac{1}{\sqrt{M^{2}+1}}\right\|_{L^{2}(\mathbb{T})}^{2} \\
& =\sum_{n \in \mathbb{Z}^{*}}\left(\left|a_{n}^{+} \gamma_{|n|}^{+}+a_{n}^{-} \gamma_{|n|}^{-}\right|^{2}+\left|a_{n}^{+} \gamma_{|n|}^{+} \frac{n^{2}}{\mu_{|n|}^{+}}+a_{n}^{-} \gamma_{|n|}^{-} \frac{n^{2}}{\mu_{|n|}^{-}}\right|^{2}\right)+\left|a_{0}^{+}\right|^{2}+\left|a_{0}^{-}\right|^{2}+2 \frac{M}{\sqrt{M^{2}+1}} \mathfrak{R}\left(a_{0}^{+} a_{0}^{-}\right) \text {. }
\end{aligned}
$$


Taking into account the fact that $\left|\gamma_{|n|}^{ \pm}\right|^{2} \leq 1,\left|\gamma_{|n|}^{ \pm}\right|^{2}\left|\frac{n^{2}}{\mu_{|n|}^{ \pm}}\right|^{2} \leq 1$ and by using the inequalities

$$
\left|z_{1}+z_{2}\right|^{2} \leq 2\left(\left|z_{1}\right|^{2}+\left|z_{2}\right|^{2}\right) ; \quad 2 \mathfrak{R}\left(z_{1} z_{2}\right) \leq\left|z_{1}\right|^{2}+\left|z_{2}\right|^{2} \quad\left(z_{1}, z_{2} \in \mathbb{C}\right),
$$

we deduce that the second inequality (16) holds for $K_{2}=4$. To obtain the first inequality in (16), the following estimate is used,

$$
\begin{aligned}
& \left|z_{1} \alpha_{1}+z_{2} \beta_{1}\right|^{2}+\left|z_{1} \alpha_{2}+z_{2} \beta_{2}\right|^{2} \\
& =\left(\alpha_{1}^{2}+\alpha_{2}^{2}\right)\left|z_{1}\right|^{2}+\left(\beta_{1}^{2}+\beta_{2}^{2}\right)\left|z_{2}\right|^{2}+2 \alpha_{1} \beta_{1} \mathfrak{R}\left(z_{1} \bar{z}_{2}\right)+2 \alpha_{2} \beta_{2} \mathfrak{R}\left(\bar{z}_{1} z_{2}\right) \\
& \geq\left(\alpha_{1}^{2}+\alpha_{2}^{2}\right)\left|z_{1}\right|^{2}+\left(\beta_{1}^{2}+\beta_{2}^{2}\right)\left|z_{2}\right|^{2}-\left|\alpha_{1} \beta_{1}\right|\left(\delta^{2}\left|z_{1}\right|^{2}+\frac{1}{\delta^{2}}\left|z_{2}\right|^{2}\right)-\left|\alpha_{2} \beta_{2}\right|\left(\delta^{2}\left|z_{1}\right|^{2}+\frac{1}{\delta^{2}}\left|z_{2}\right|^{2}\right) \\
& =\left(\alpha_{1}^{2}+\alpha_{2}^{2}-\left|\alpha_{1} \beta_{1}\right| \delta^{2}-\left|\alpha_{2} \beta_{2}\right| \delta^{2}\right)\left|z_{1}\right|^{2}+\left(\beta_{1}^{2}+\beta_{2}^{2}-\left|\alpha_{1} \beta_{1}\right| \frac{1}{\delta^{2}}-\left|\alpha_{2} \beta_{2}\right| \frac{1}{\delta^{2}}\right)\left|z_{2}\right|^{2},
\end{aligned}
$$

for any $z_{1}, z_{2} \in \mathbb{C}, \alpha_{1}, \alpha_{2}, \beta_{1}, \beta_{2} \in \mathbb{R}$ and $\delta>0$ such that

$$
\delta^{2} \in\left(\frac{\left|\alpha_{1} \beta_{1}\right|+\left|\alpha_{2} \beta_{2}\right|}{\beta_{1}^{2}+\beta_{2}^{2}}, \frac{\alpha_{1}^{2}+\alpha_{2}^{2}}{\left|\alpha_{1} \beta_{1}\right|+\left|\alpha_{2} \beta_{2}\right|}\right) .
$$

In the above inequality if we consider $z_{1}=a_{n}^{+}, z_{2}=a_{n}^{-}, \alpha_{1}=\gamma_{|n|}^{+}, \beta_{1}=\gamma_{|n|}^{-}, \alpha_{2}=\gamma_{|n|}^{+} \frac{n^{2}}{\mu_{|n|}^{+}}$and $\beta_{2}=\gamma_{|n|}^{-} \frac{n^{2}}{\mu_{|n|}^{-}}$it follows that

$$
\begin{aligned}
& \left|a_{n}^{+} \gamma_{|n|}^{+}+a_{n}^{-} \gamma_{|n|}^{-}\right|^{2}+\left|a_{n}^{+} \gamma_{|n|}^{+} \frac{n^{2}}{\mu_{|n|}^{+}}+a_{n}^{-} \gamma_{|n|}^{-} \frac{n^{2}}{\mu_{|n|}^{-}}\right|^{2} \\
& \geq[\left.\underbrace{\left(\gamma_{|n|}^{+}\right)^{2}+\left(\gamma_{|n|}^{+} \frac{n^{2}}{\mu_{|n|}^{+}}\right)^{2}-\left|\gamma_{|n|}^{+} \gamma_{|n|}^{-}\right| \delta_{n}^{2}-\left|\gamma_{|n|}^{+} \frac{n^{2}}{\mu_{|n|}^{+}} \gamma_{|n|}^{-} \frac{n^{2}}{\mu_{|n|}^{-}}\right| \delta_{n}^{2}}_{:=C_{n}^{1}}|| a_{n}^{+}\right|^{2} \\
& +[\underbrace{\left(\gamma_{|n|}^{-}\right)^{2}+\left(\gamma_{|n|}^{-} \frac{n^{2}}{\mu_{|n|}^{-}}\right)^{2}-\left|\gamma_{|n|}^{+} \gamma_{|n|}^{-}\right| \frac{1}{\delta_{n}^{2}}-\left|\gamma_{|n|}^{+} \frac{n^{2}}{\mu_{|n|}^{+}} \gamma_{|n|}^{-} \frac{n^{2}}{\mu_{|n|}^{-}}\right| \frac{1}{\delta_{n}^{2}}}_{:=C_{n}^{2}}]\left|a_{n}^{-}\right|^{2} .
\end{aligned}
$$

We remark that $C_{n}^{1}, C_{n}^{2}>0$ for every $\delta_{n}>0$ such that

$$
\delta_{n}^{2} \in\left(\frac{\left|\gamma_{|n|}^{+} \gamma_{|n|}^{-}\right|+\left|\gamma_{|n|}^{+} \frac{n^{2}}{\mu_{n \mid l}^{+}} \gamma_{|n|}^{-} \frac{n^{2}}{\mu_{|n|}^{-}}\right|}{\left(\gamma_{|n|}^{-}\right)^{2}+\left(\gamma_{|n|}^{-} \frac{n^{2}}{\mu_{|n|}}\right)^{2}}, \frac{\left(\gamma_{|n|}^{+}\right)^{2}+\left(\gamma_{|n|}^{+} \frac{n^{2}}{\mu_{|n|}^{+}}\right)^{2}}{\left|\gamma_{|n|}^{+} \gamma_{|n|}^{-}\right|+\mid \gamma_{|n|}^{+} \frac{n^{2}}{\mu_{|n|}^{+}} \gamma_{|n|}^{-} \frac{n^{2}}{\mu_{|n|}}}\right) .
$$

If we choose

$$
\delta_{n}^{2}=\frac{1}{2}\left(\frac{\left|\gamma_{|n|}^{+} \gamma_{|n|}^{-}\right|+\left|\gamma_{|n|}^{+} \frac{n^{2}}{\mu_{|n|}^{+}} \gamma_{|n|}^{-} \frac{n^{2}}{\mu_{|n| n}}\right|}{\left(\gamma_{|n|}^{-}\right)^{2}+\left(\gamma_{|n|}^{-} \frac{n^{2}}{\mu_{|n|}^{2}}\right)^{2}}+\frac{\left(\gamma_{|n|}^{+}\right)^{2}+\left(\gamma_{|n|}^{+} \frac{n^{2}}{\mu_{|n|}^{+}}\right)^{2}}{\left|\gamma_{|n|}^{+} \gamma_{|n|}^{-}\right|+\mid \gamma_{|n|}^{+} \frac{n^{2}}{\mu_{|n|}^{+}} \gamma_{|n| \frac{n^{2}}{\mu_{|n|}}}^{-}}\right),
$$


and we take into account the fact that

$$
\lim _{n \rightarrow \infty} \gamma_{|n|}^{+}=\frac{1}{\sqrt{2}}, \quad \lim _{n \rightarrow \infty} \gamma_{|n|}^{-}=0, \quad \lim _{n \rightarrow \infty} \gamma_{|n|}^{+} \frac{n^{2}}{\mu_{|n|}^{+}}=\frac{1}{\sqrt{2}}, \quad \lim _{n \rightarrow \infty} \gamma_{|n|}^{-} \frac{n^{2}}{\mu_{|n|}^{-}}=-1,
$$

we obtain

$$
\lim _{n \rightarrow \infty} \delta_{n}^{2}=\frac{3}{2 \sqrt{2}}, \quad \lim _{n \rightarrow \infty} C_{n}^{1}=\frac{1}{4}, \quad \lim _{n \rightarrow \infty} C_{n}^{2}=\frac{1}{3} .
$$

From (19) it follows that there exists $N_{0} \in \mathbb{N}^{*}$ such that, for any $|n|>N_{0}$, we have that

$$
C_{n}^{1}>\frac{1}{8}, \quad C_{n}^{2}>\frac{1}{6}
$$

From the above estimate it follows that

$$
\left|a_{n}^{+} \gamma_{|n|}^{+}+a_{n}^{-} \gamma_{|n|}^{-}\right|^{2}+\left|a_{n}^{+} \gamma_{|n|}^{+} \frac{n^{2}}{\mu_{|n|}^{+}}+a_{n}^{-} \gamma_{|n|}^{-} \frac{n^{2}}{\mu_{|n|}^{-}}\right|^{2} \geq \frac{1}{8}\left(\left|a_{n}^{+}\right|^{2}+\left|a_{n}^{-}\right|^{2}\right) \quad\left(|n|>N_{0}\right)
$$

and we deduce that

$$
\sum_{n \in \mathbb{Z}^{*}}\left(\left|a_{n}^{+} \gamma_{|n|}^{+}+a_{n}^{-} \gamma_{|n|}^{-}\right|^{2}+\left|a_{n}^{+} \gamma_{|n|}^{+} \frac{n^{2}}{\mu_{|n|}^{+}}+a_{n}^{-} \gamma_{|n|}^{-} \frac{n^{2}}{\mu_{|n|}^{-}}\right|^{2}\right) \geq \min \left\{\frac{1}{8}, \min _{1 \leq|n| \leq N_{0}}\left\{C_{n}^{1}, C_{n}^{2}\right\}\right\} \sum_{n \in \mathbb{Z}^{*}}\left|a_{n}^{ \pm}\right|^{2}
$$

On the other hand, we have that

$$
\left|a_{0}^{+}\right|^{2}+\left|a_{0}^{-}\right|^{2}+2 \frac{M}{\sqrt{M^{2}+1}} \mathfrak{R}\left(a_{0}^{+} a_{0}^{-}\right) \geq\left(1-\frac{M}{\sqrt{M^{2}+1}}\right)\left|a_{0}^{+}\right|^{2}+\left(1-\frac{M}{\sqrt{M^{2}+1}}\right)\left|a_{0}^{-}\right|^{2} .
$$

From (17), (18), (20) and (21) we deduce that the left inequality (16) holds for

$$
K_{1}=\min \left\{1-\frac{M}{\sqrt{M^{2}+1}}, \frac{1}{8}, \min _{1 \leq|n| \leq N_{0}}\left\{C_{n}^{1}, C_{n}^{2}\right\}\right\},
$$

and the proof is complete.

Remark 2.2 In Theorem 2.1 we show that $\left(\gamma_{|n|}^{ \pm} \Phi_{n}^{ \pm}\right)_{n \in \mathbb{Z}^{*}} \cup\left\{\Phi_{0}^{+}, \frac{M}{\sqrt{M^{2}+1}} \Phi_{0}^{-}\right\}$is a Riesz basis in the spaces $\left(L^{2}(\mathbb{T})\right)^{2}$. Thus, $\left(\begin{array}{c}u^{0} \\ z^{0}\end{array}\right) \in\left(L^{2}(\mathbb{T})\right)^{2}$ if and only if there exists a unique sequence of coefficients $\left(a_{n}^{0, \pm}\right)_{n \in \mathbb{Z}}$ such that

$$
\left(a_{n}^{0, \pm}\right)_{n \in \mathbb{Z}} \in \ell^{2}
$$

and

$$
\left(\begin{array}{c}
u^{0} \\
z^{0}
\end{array}\right)=\sum_{n \in \mathbb{Z}^{*}} a_{n}^{0, \pm} \gamma_{|n|}^{ \pm} \Phi_{n}^{ \pm}+a_{0}^{0,+} \Phi_{0}^{+}+a_{0}^{0,-} \frac{M}{\sqrt{M^{2}+1}} \Phi_{0}^{-} .
$$

Moreover, we have that $F \in L^{2}\left((0, T) ;\left(L^{2}(\mathbb{T})\right)^{2}\right)$ if and only if, for a.e. $t \in(0, T)$, there exists a unique sequence $\left(\widetilde{f_{n}^{ \pm}}(t)\right)_{n \in \mathbb{Z}} \in \ell^{2}$ such that

$$
\sum_{n \in \mathbb{Z}} \int_{0}^{T}\left|\widetilde{f}_{n}^{ \pm}(t)\right|^{2} \mathrm{dt}<\infty
$$


and

$$
F(t)=\sum_{n \in \mathbb{Z}^{*}}{\widetilde{f_{n}^{ \pm}}}^{ \pm}(t) \gamma_{|n|}^{ \pm} \Phi_{n}^{ \pm}+{\widetilde{f_{0}^{+}}}^{+}(t) \Phi_{0}^{+}+{\widetilde{f_{0}^{-}}}_{0}(t) \frac{M}{\sqrt{M^{2}+1}} \Phi_{0}^{-} .
$$

On the other hand, given $f \in L^{2}\left((0, T) ; L^{2}(\mathbb{T})\right)$ such that

$$
f(t, x)=\sum_{n \in \mathbb{Z}} f_{n}(t) e^{i n x}
$$

we have that $F=\left(\begin{array}{l}f \\ 0\end{array}\right)$ is given by (25), where the Fourier coefficients have the particular form

$$
\left\{\begin{array}{l}
\widetilde{f}_{n}^{ \pm}(t)=\frac{\mu_{|n|}^{ \pm} f_{n}(t)}{\left.\gamma_{|n|}^{ \pm} \mid \mu_{|n|}^{ \pm}-\mu_{|n|}^{\mp}\right)} \quad(n \neq 0), \\
\widetilde{f}_{0}^{-}(t)=0, \quad \widetilde{f}_{0}^{+}(t)=f_{0}(t) .
\end{array}\right.
$$

\section{Well posedness results}

In this section we study the existence, uniqueness and regularity properties of the solution of (4). We shall introduce two concepts of solution: weak and classical.

Definition 3.1 $\left(\begin{array}{c}u \\ z\end{array}\right) \in C^{1}\left([0, T] ;\left(L^{2}(\mathbb{T})\right)^{2}\right) \cap C([0, T] ; D(\mathcal{A}))$ is a classical solution of (4) in $\left(L^{2}(\mathbb{T})\right)^{2}$ if satisfies the equation for each $t \in[0, T]$ and the initial conditions.

Definition 3.2 $\left(\begin{array}{c}u \\ z\end{array}\right) \in C\left([0, T] ;\left(L^{2}(\mathbb{T})\right)^{2}\right)$ is a weak solution of (4) if verifies the following variational equality

$$
\begin{aligned}
& -\int_{0}^{t} \int_{\mathbb{T}} u(s, x) \varphi_{s}(s, x) \mathrm{dx} \mathrm{ds}-\int_{0}^{t} \int_{\mathbb{T}} u(s, x) \varphi_{x x}(s, x) \mathrm{dx} \mathrm{ds}+M \int_{0}^{t} \int_{\mathbb{T}} z(s, x) \varphi(s, x) \mathrm{dx} \mathrm{d} s \\
& +\int_{\mathbb{T}} u(t, x) \varphi(t, x) \mathrm{dx}-\int_{\mathbb{T}} u^{0}(x) \varphi(0, x) \mathrm{d} \mathrm{x}-\int_{0}^{t} \int_{\mathbb{T}} z(s, x) \psi_{s}(s, x) \mathrm{dx} \mathrm{ds} \\
& -\int_{0}^{t} \int_{\mathbb{T}} u(s, x) \psi_{x x}(s, x) \mathrm{dxd}+\int_{\mathbb{T}} z(t, x) \psi(t, x) \mathrm{dx}-\int_{\mathbb{T}} z^{0}(x) \psi(0, x) \mathrm{dx} \\
& =\int_{0}^{t} \int_{\mathbb{T}} f(s, x) \varphi(s, x) \mathrm{dxds},
\end{aligned}
$$

for any $\varphi, \psi \in C^{1}\left([0, T] ; H^{2}(\mathbb{T})\right)$ and $t \in[0, T]$.

Next, we study the solutions of the following system

$$
\left(\begin{array}{l}
u \\
z
\end{array}\right)_{t}+\mathcal{A}\left(\begin{array}{l}
u \\
z
\end{array}\right)=F, \quad\left(\begin{array}{l}
u \\
z
\end{array}\right)(0)=\left(\begin{array}{c}
u^{0} \\
z^{0}
\end{array}\right),
$$

where $\mathcal{A}$ is the operator given by (5) and $F \in L^{2}\left((0, T) ; L^{2}(\mathbb{T})\right)$.

The first result gives the simplest solutions of (29). 
Proposition 3.1 Let $n \in \mathbb{Z}, a_{n}^{0, \pm} \in \mathbb{C}$ and $\widetilde{f_{n}^{ \pm}} \in L^{2}(0, T)$. If $n \neq 0$, then the unique solution of (29) with $F=\widetilde{f_{n}^{ \pm}}(t) \Phi_{n}^{ \pm}$and $\left(\begin{array}{c}u^{0} \\ z^{0}\end{array}\right)=a_{n}^{0, \pm} \Phi_{n}^{ \pm}$is given by

$$
\left(\begin{array}{c}
u \\
z
\end{array}\right)(t)=\left(a_{n}^{0, \pm} e^{-\mu_{|n|}^{ \pm} t}+\int_{0}^{t} e^{-\mu_{|n|}^{ \pm}(t-s)} \widetilde{f}_{n}^{ \pm}(s) \mathrm{ds}\right) \Phi_{n}^{ \pm} .
$$

On the other hand, if $F=\widetilde{f}_{0}^{+}(t) \Phi_{0}^{+}$and $\left(\begin{array}{c}u^{0} \\ z^{0}\end{array}\right)=a_{0}^{0,+} \Phi_{0}^{+}+a_{0}^{0,-} \Phi_{0}^{-}$, then the corresponding solution of (29) is given by

$$
\left(\begin{array}{c}
u \\
z
\end{array}\right)(t)=\left(a_{0}^{0,+}+\int_{0}^{t} \widetilde{f_{0}^{+}}(s) \mathrm{ds}-a_{0}^{0,-} t\right) \Phi_{0}^{+}+a_{0}^{0,-} \Phi_{0}^{-} .
$$

Proof. Firstly, we consider the case $n \neq 0$. We look for a solution of (29) of the form $\left(\begin{array}{c}u \\ z\end{array}\right)(t)=a_{n}^{ \pm}(t) \Phi_{n}^{ \pm}$. We deduce that

$$
\left(a_{n}^{ \pm}\right)^{\prime}(t)+\mu_{|n|}^{ \pm} a_{n}^{ \pm}(t)=\widetilde{f}_{n}^{ \pm}(t) .
$$

Moreover, since $\left(\begin{array}{c}u^{0} \\ z^{0}\end{array}\right)=\left(\begin{array}{c}u \\ z\end{array}\right)(0)$ we obtain the initial conditions

$$
a_{n}^{ \pm}(0)=a_{n}^{0, \pm} \quad\left(n \in \mathbb{Z}^{*}\right) .
$$

Solving (32) with initial conditions (33) it follows that

$$
a_{n}^{ \pm}(t)=a_{n}^{0, \pm} e^{-\mu_{|n|}^{ \pm} t}+\int_{0}^{t} e^{-\mu_{|n|}^{ \pm}(t-s)} \widetilde{f_{n}^{ \pm}}(s) \mathrm{ds}
$$

and (30) holds.

On the other hand, if $n=0$ we look for a solution of (29) of the form $\left(\begin{array}{c}u \\ z\end{array}\right)(t)=a_{0}^{+}(t) \Phi_{0}^{+}+$ $a_{0}^{-}(t) \Phi_{0}^{-}$. It follows that

$$
\left\{\begin{array}{l}
\left(a_{0}^{+}\right)^{\prime}(t)+a_{0}^{-}(t)=\widetilde{f_{0}^{+}}(t) \\
\left(a_{0}^{-}\right)^{\prime}(t)=0
\end{array}\right.
$$

Since $\left(\begin{array}{c}u^{0} \\ z^{0}\end{array}\right)=\left(\begin{array}{c}u \\ z\end{array}\right)(0)$ we obtain the initial conditions

$$
a_{0}^{ \pm}(0)=a_{0}^{0, \pm} .
$$

Solving (34) with initial conditions (35) we deduce that

$$
\left\{\begin{array}{l}
a_{0}^{+}(t)=a_{0}^{0,+}+\int_{0}^{t} \widetilde{f_{0}^{+}}(s) \mathrm{ds}-a_{0}^{0,-} t \\
a_{0}^{-}(t)=a_{0}^{0,-}
\end{array}\right.
$$

and (31) holds.

Remark 3.1 Let $\left(\begin{array}{c}u^{0} \\ z^{0}\end{array}\right) \in\left(L^{2}(\mathbb{T})\right)^{2}$ of the form (23) and let $f \in L^{2}\left((0, T) ; L^{2}(\mathbb{T})\right)$ of the form (26). It follows that the expansion of $\left(\begin{array}{l}f \\ 0\end{array}\right)$ is given by (25) with the Fourier coefficients (27). 
By taking into account the previous proposition, the corresponding solution of the problem (29) with $F=\left(\begin{array}{l}f \\ 0\end{array}\right)$ should be given by the Fourier expansion

$$
\left(\begin{array}{c}
u \\
z
\end{array}\right)(t)=\sum_{n \in \mathbb{Z}^{*}} a_{n}^{ \pm}(t) \gamma_{|n|}^{ \pm} \Phi_{n}^{ \pm}+a_{0}^{+}(t) \Phi_{0}^{+}+a_{0}^{-}(t) \frac{M}{\sqrt{M^{2}+1}} \Phi_{0}^{-},
$$

where

$$
\left\{\begin{array}{l}
a_{n}^{ \pm}(t)=a_{n}^{0, \pm} e^{-\mu_{|n|}^{ \pm} t}+\int_{0}^{t} e^{-\mu_{|n|}^{ \pm}(t-s)} \widetilde{f_{n}^{ \pm}}(s) \mathrm{ds} \quad(n \neq 0), \\
a_{0}^{-}(t)=a_{0}^{0,-}, \\
a_{0}^{+}(t)=a_{0}^{0,+}+\int_{0}^{t} \widetilde{f_{0}^{+}}(s) \mathrm{ds}-a_{0}^{0,-} \frac{M}{\sqrt{M^{2}+1}} t .
\end{array}\right.
$$

Moreover, the coefficients $\left(a_{n}^{ \pm}(t)\right)_{n \in \mathbb{Z}}$ given by (37) are uniquely determined.

The properties of (36) will be studied in the following two theorems.

Remark 3.2 We have that $\left(\begin{array}{c}u^{0} \\ z^{0}\end{array}\right) \in\left(L^{2}(\mathbb{T})\right)^{2}$ given by (23) belongs to $D(\mathcal{A})$ if and only if

$$
\left(a_{n}^{0, \pm} \mu_{|n|}^{ \pm}\right)_{n \in \mathbb{Z}^{*}} \in \ell^{2}
$$

Moreover, if $\left(\begin{array}{l}f \\ 0\end{array}\right) \in H^{1}\left((0, T) ;\left(L^{2}(\mathbb{T})\right)^{2}\right)$ and $f$ is given by (26), then we deduce that

$$
\sum_{n \in \mathbb{Z}} \int_{0}^{T}\left|\left(\widetilde{f_{n}^{ \pm}}\right)^{\prime}(t)\right|^{2} \mathrm{dt}<\infty
$$

where $\widetilde{f_{n}^{ \pm}}$are given by (27).

In the next theorem we study the weak solution of (4).

Theorem 3.1 Given $\left(\begin{array}{c}u^{0} \\ z^{0}\end{array}\right) \in\left(L^{2}(\mathbb{T})\right)^{2}$ and $f \in L^{2}\left((0, T) ; L^{2}(\mathbb{T})\right)$, then (4) has a unique weak solution.

Proof. Let $\left(\begin{array}{c}u \\ z\end{array}\right)$ given by (36). We show that $\left(\begin{array}{c}u \\ z\end{array}\right)$ is the unique weak solution of (4).

Firstly, we prove that $\left(\begin{array}{c}u \\ z\end{array}\right)(t) \in\left(L^{2}(\mathbb{T})\right)^{2}$, for each $t \in[0, T]$. Since $\left(\gamma_{|n|}^{ \pm} \Phi_{n}^{ \pm}\right)_{n \in \mathbb{Z}^{*}} U$ $\left\{\Phi_{0}^{+}, \frac{M}{\sqrt{M^{2}+1}} \Phi_{0}^{-}\right\}$is a Riesz basis in the spaces $\left(L^{2}(\mathbb{T})\right)^{2}$ it follows that $\sum_{n \in \mathbb{Z}^{*}} a_{n}^{ \pm}(t) \gamma_{|n|}^{ \pm} \Phi_{n}^{ \pm}+$ $a_{0}^{+}(t) \Phi_{0}^{+}+a_{0}^{-}(t) \frac{M}{\sqrt{M^{2}+1}} \Phi_{0}^{-} \in\left(L^{2}(\mathbb{T})\right)^{2}$, if and only if, $\left(a_{n}^{ \pm}(t)\right)_{n \in \mathbb{Z}} \in \ell^{2}$, for any $t \in[0, T]$. Indeed, by the form (37) of the coefficients $a_{n}^{ \pm}(t)$, we have that

$$
\begin{aligned}
\sum_{n \in \mathbb{Z}}\left|a_{n}^{ \pm}(t)\right|^{2} & \leq 2 \sum_{n \in \mathbb{Z}^{*}}\left|a_{n}^{0, \pm} e^{-\mu_{|n|}^{ \pm} t}\right|^{2}+2 \sum_{n \in \mathbb{Z}^{*}}\left|\int_{0}^{t} e^{-\mu_{|n|}^{ \pm}(t-s)} \widetilde{f}_{n}^{ \pm}(s) \mathrm{ds}\right|^{2} \\
& +(3 T+1)\left|a_{0}^{0,-}\right|^{2}+3\left|a_{0}^{0,+}\right|^{2}+3\left|\int_{0}^{T} \widetilde{f_{0}^{+}}(s) \mathrm{ds}\right|^{2} \\
& \leq C\left(\sum_{n \in \mathbb{Z}}\left|a_{n}^{0, \pm}\right|^{2}+\sum_{n \in \mathbb{Z}} \int_{0}^{T}\left|\widetilde{f}_{n}^{ \pm}(s)\right|^{2} \mathrm{ds}\right)
\end{aligned}
$$


From (22) and (24), it follows that the last two series are convergent. By using the Weierstrass test we deduce that $\left(\begin{array}{c}u \\ z\end{array}\right) \in C\left([0, T] ;\left(L^{2}(\mathbb{T})\right)^{2}\right)$.

For $N \in \mathbb{N}^{*}$, we consider

$$
\left(\begin{array}{c}
u_{N}^{0} \\
z_{N}^{0}
\end{array}\right)=\sum_{1 \leq|n| \leq N} a_{n}^{0, \pm} \gamma_{|n|}^{ \pm} \Phi_{n}^{ \pm}+a_{0}^{0,+} \Phi_{0}^{+}+a_{0}^{0,-} \frac{M}{\sqrt{M^{2}+1}} \Phi_{0}^{-},
$$

and

$$
f_{N}(t)=\sum_{0 \leq|n| \leq N} f_{n}(t) e^{i n x}
$$

We have that

$$
\left(\begin{array}{c}
f_{N} \\
0
\end{array}\right)=\sum_{1 \leq|n| \leq N} \widetilde{f_{n}^{ \pm}}(t) \gamma_{|n|}^{ \pm} \Phi_{n}^{ \pm}+\widetilde{f}_{0}^{+}(t) \Phi_{0}^{+}+\widetilde{f}_{0}^{-}(t) \frac{M}{\sqrt{M^{2}+1}} \Phi_{0}^{-},
$$

where the Fourier coefficients $\left(\widetilde{f}_{n}^{ \pm}(t)\right)_{0 \leq n \mid \leq N}$ are given by (27). (40) is

The corresponding solution of the problem (29) with $F=\left(\begin{array}{c}f_{N} \\ 0\end{array}\right)$ and $\left(\begin{array}{c}u_{N}^{0} \\ z_{N}^{0}\end{array}\right)$ given by

$$
\left(\begin{array}{c}
u_{N} \\
z_{N}
\end{array}\right)(t)=\sum_{1 \leq|n| \leq N} a_{n}^{ \pm}(t) \gamma_{|n|}^{ \pm} \Phi_{n}^{ \pm}+a_{0}^{+}(t) \Phi_{0}^{+}+a_{0}^{-}(t) \frac{M}{\sqrt{M^{2}+1}} \Phi_{0}^{-},
$$

where the Fourier coefficients $\left(a_{n}^{ \pm}(t)\right)_{0 \leq|n| \leq N}$ are given by (37).

Taking into account the form of $\left(\begin{array}{c}u_{N} \\ z_{N}\end{array}\right)$ given by (43), it follows that the relation

$$
\begin{aligned}
& -\int_{0}^{t} \int_{\mathbb{T}} u_{N}(s, x) \varphi_{s}(s, x) \mathrm{dxds}-\int_{0}^{t} \int_{\mathbb{T}} u_{N}(s, x) \varphi_{x x}(s, x) \mathrm{dxds}+M \int_{0}^{t} \int_{\mathbb{T}} z_{N}(s, x) \varphi(s, x) \mathrm{dx} \mathrm{ds} \\
& +\int_{\mathbb{T}} u_{N}(t, x) \varphi(t, x) \mathrm{dx}-\int_{\mathbb{T}} u_{N}^{0}(x) \varphi(0, x) \mathrm{dx}-\int_{0}^{t} \int_{\mathbb{T}} z_{N}(s, x) \psi_{s}(s, x) \mathrm{dx} \mathrm{ds} \\
& -\int_{0}^{t} \int_{\mathbb{T}} u_{N}(s, x) \psi_{x x}(s, x) \mathrm{dx} \mathrm{ds}+\int_{\mathbb{T}} z_{N}(t, x) \psi(t, x) \mathrm{dx}-\int_{\mathbb{T}} z_{N}^{0}(x) \psi(0, x) \mathrm{d} \mathrm{x} \\
& =\int_{0}^{t} \int_{\mathbb{T}} f_{N}(s, x) \varphi(s, x) \mathrm{dx} \mathrm{ds},
\end{aligned}
$$

holds, for any $\varphi, \psi \in C^{1}\left([0, T] ; H^{2}(\mathbb{T})\right)$ and $t \in[0, T]$. that

$$
\text { Since }\left(\begin{array}{c}
u \\
z
\end{array}\right) \in C\left([0, T] ;\left(L^{2}(\mathbb{T})\right)^{2}\right),\left(\begin{array}{c}
u^{0} \\
z^{0}
\end{array}\right) \in\left(L^{2}(\mathbb{T})\right)^{2} \text { and } f \in L^{2}\left((0, T) ; L^{2}(\mathbb{T})\right) \text { we have }
$$

$$
\begin{gathered}
\left(\begin{array}{c}
u_{N} \\
z_{N}
\end{array}\right) \rightarrow\left(\begin{array}{c}
u \\
z
\end{array}\right) \text { in } C\left([0, T] ;\left(L^{2}(\mathbb{T})\right)^{2}\right), \text { when } N \rightarrow \infty, \\
\left(\begin{array}{c}
u_{N}^{0} \\
z_{N}^{0}
\end{array}\right) \rightarrow\left(\begin{array}{c}
u^{0} \\
z^{0}
\end{array}\right) \text { in }\left(L^{2}(\mathbb{T})\right)^{2}, \text { when } N \rightarrow \infty, \\
f_{N} \rightarrow f \text { in } L^{2}\left((0, T) ; L^{2}(\mathbb{T})\right), \text { when } N \rightarrow \infty
\end{gathered}
$$

Taking into account the last three convergences and passing to the limit in (44), when $N \rightarrow \infty$, we deduce that $\left(\begin{array}{l}u \\ z\end{array}\right)$ given by (36) verifies (28), for any $\varphi, \psi \in C^{1}\left([0, T] ; H^{2}(\mathbb{T})\right)$ and $t \in[0, T]$. 
The uniqueness of the solution follows immediately from Remark 3.1, since the coefficients $\left(a_{n}^{ \pm}(t)\right)_{n \in \mathbb{Z}}$ from (36) are uniquely determined, and the proof is complete.

Now, we pass to study the classical solution of (4).

\section{Theorem 3.2 Given}

$$
\begin{aligned}
& \left(\begin{array}{c}
u^{0} \\
z^{0}
\end{array}\right) \in D(\mathcal{A}), \\
& f \in H^{1}\left((0, T) ; L^{2}(\mathbb{T})\right),
\end{aligned}
$$

then (4) has a unique classical solution in $\left(L^{2}(\mathbb{T})\right)^{2}$.

Proof. Let $\left(\begin{array}{c}u \\ z\end{array}\right)$ given by (36). We show that $\left(\begin{array}{l}u \\ z\end{array}\right)$ is the unique classical solution of (4).

Firstly, we prove that $\left(\begin{array}{c}u \\ z\end{array}\right) \in C([0, T] ; D(\mathcal{A}))$. We remark that, formally,

$$
\mathcal{A}\left(\begin{array}{c}
u \\
z
\end{array}\right)(t)=\sum_{n \in \mathbb{Z}^{*}} a_{n}^{ \pm}(t) \mu_{|n|}^{ \pm} \gamma_{|n|}^{ \pm} \Phi_{n}^{ \pm}
$$

We prove that the right hand side term is well defined for each $t \in[0, T]$ and represents a function from $C\left([0, T] ;\left(L^{2}(\mathbb{T})\right)^{2}\right)$. We have that

$$
\begin{aligned}
a_{n}^{ \pm}(t) \mu_{|n|}^{ \pm} & =\mu_{|n|}^{ \pm} a_{n}^{0, \pm} e^{-\mu_{|n|}^{ \pm} t}+\mu_{|n|}^{ \pm} \int_{0}^{t} e^{-\mu_{|n|}^{ \pm}(t-s)} \widetilde{f_{n}^{ \pm}}(s) \mathrm{ds} \\
& =\mu_{|n|}^{ \pm} a_{n}^{0, \pm} e^{-\mu_{|n|}^{ \pm} t}+e^{-\mu_{|n|}^{ \pm} t} \int_{0}^{t}\left(e^{\mu_{|n|}^{ \pm} s}\right)^{\prime} \widetilde{f_{n}^{ \pm}}(s) \mathrm{ds} \\
& =\mu_{|n|}^{ \pm} a_{n}^{0, \pm} e^{-\mu_{|n|}^{ \pm} t}+\widetilde{f_{n}^{ \pm}}(t)-e^{-\mu_{|n|}^{ \pm} t} \widetilde{f_{n}^{ \pm}}(0)-e^{-\mu_{|n|}^{ \pm} t} \int_{0}^{t} e^{\mu_{|n|}^{ \pm} s}\left(\widetilde{f_{n}^{ \pm}}\right)^{\prime}(s) \mathrm{d} s
\end{aligned}
$$

From the above relations, using the hypothesis $\left(\widetilde{f}_{n}^{ \pm}(t)\right)_{n \in \mathbb{Z}^{*}} \in \ell^{2}$ and the fact that by (45) and (46) the conditions (38) and (39) are fulfilled, then with similar arguments used in the proof of Theorem 3.1 we deduce that the right hand side term is well defined and represents a function from $C\left([0, T] ;\left(L^{2}(\mathbb{T})\right)^{2}\right)$. Consequently, $\left(\begin{array}{c}u \\ z\end{array}\right) \in C([0, T] ; D(\mathcal{A}))$.

Next we prove that $\left(\begin{array}{c}u \\ z\end{array}\right) \in C^{1}\left([0, T] ;\left(L^{2}(\mathbb{T})\right)^{2}\right)$. For any $k \geq 1$, we consider

$$
g_{k}(t)=\sum_{1 \leq|n| \leq k} a_{n}^{ \pm}(t) \gamma_{|n|}^{ \pm} \Phi_{n}^{ \pm}+a_{0}^{+}(t) \Phi_{0}^{+}+a_{0}^{-}(t) \frac{M}{\sqrt{M^{2}+1}} \Phi_{0}^{-} \quad(t \in[0, T]) .
$$

According to the classical results concerning the derivation of a series (see for instance [4]) we have to prove that:

1. $g_{k} \in C^{1}\left([0, T] ;\left(L^{2}(\mathbb{T})^{2}\right)\right.$;

2. $g_{k}$ converges pointwise to $g$ in $[0, T]$;

3. $\left(g_{k}^{\prime}\right)_{k}$ is uniformly convergent on $[0, T]$. 
We have that

$$
\begin{aligned}
g_{k}^{\prime}(t) & =\sum_{1 \leq|n| \leq k}\left[-a_{n}^{0, \pm} \mu_{|n|}^{ \pm} e^{-\mu_{|n|}^{ \pm} t}-\mu_{|n|}^{ \pm} e^{-\mu_{|n|}^{ \pm} t} \int_{0}^{t} e^{\mu_{|n|}^{ \pm} s} \widetilde{f_{n}^{ \pm}}(s) \mathrm{ds}+\widetilde{f}_{n}^{ \pm}(t)\right] \gamma_{|n|}^{ \pm} \Phi_{n}^{ \pm} \\
& +\left(\widetilde{f_{0}^{+}}(t)-a_{0}^{0,-} \frac{M}{\sqrt{M^{2}+1}}\right) \Phi_{0}^{+} \\
& =\sum_{1 \leq|n| \leq k}\left[-a_{n}^{0, \pm} \mu_{|n|}^{ \pm} e^{-\mu_{|n|}^{ \pm} t}-e^{-\mu_{|n|}^{ \pm} t} \int_{0}^{t}\left(e^{\mu_{|n|}^{ \pm} s}\right)^{\prime} \widetilde{f_{n}^{ \pm}}(s) \mathrm{ds}+\widetilde{f_{n}^{ \pm}}(t)\right] \gamma_{|n|}^{ \pm} \Phi_{n}^{ \pm} \\
& +\left(\widetilde{f}_{0}^{+}(t)-a_{0}^{0,-} \frac{M}{\sqrt{M^{2}+1}}\right) \Phi_{0}^{+} \\
& =\sum_{1 \leq|n| \leq k}\left[-a_{n}^{0, \pm} \mu_{|n|}^{ \pm} e^{-\mu_{|n|}^{ \pm} t}+e^{-\mu_{|n|}^{ \pm} t} \widetilde{f}_{n}^{ \pm}(0)+e^{-\mu_{|n|}^{ \pm} t} \int_{0}^{t} e^{\mu_{|n|}^{ \pm} s}\left(\widetilde{f_{n}^{ \pm}}\right)^{\prime}(s) \mathrm{ds}\right] \gamma_{|n|}^{ \pm} \Phi_{n}^{ \pm} \\
& +\left(\widetilde{f_{0}^{+}}(t)-a_{0}^{0,-} \frac{M}{\sqrt{M^{2}+1}}\right) \Phi_{0}^{+} .
\end{aligned}
$$

Since $\widetilde{f_{n}^{ \pm}} \in H^{1}(0, T)$, it follows that $g_{k} \in C^{1}\left([0, T] ;\left(L^{2}(\mathbb{T})\right)^{2}\right)$ and the first assumption 1 is verified. The second assumption 2 follows immediately since $g_{k}(t)$ converge to $g(t):=\left(\begin{array}{c}u \\ z\end{array}\right)(t)$, the solution given by (36) which is well defined. It remains to verify the last assumption 3. We remark that

$$
\begin{gathered}
\left|-a_{n}^{0, \pm} \mu_{|n|}^{ \pm} e^{-\mu_{|n|}^{ \pm} t}+e^{-\mu_{|n|}^{ \pm} t} \widetilde{f_{n}^{ \pm}}(0)+e^{-\mu_{|n|}^{ \pm} t} \int_{0}^{t} e^{\mu_{|n|}^{ \pm} s}\left(\widetilde{f_{n}^{ \pm}}\right)^{\prime}(s) \mathrm{ds}\right|^{2} \\
\leq C\left(\left|a_{n}^{0, \pm} \mu_{|n|}^{ \pm}\right|^{2}+\left|\widetilde{f_{n}^{ \pm}}(0)\right|^{2}+\int_{0}^{T}\left|\left(\widetilde{f}_{n}^{ \pm}\right)^{\prime}(s)\right|^{2} \mathrm{ds}\right) .
\end{gathered}
$$

By taking into account (38), (39) and the fact that $\left(\widetilde{f}_{n}^{ \pm}(t)\right)_{n \in \mathbb{Z}} \in \ell^{2}$ for each $t \in[0, T]$, with similar arguments as in the proof of Theorem 3.1, it follows that the sequence $\left(g_{k}^{\prime}\right)_{k \geq 1}$ converges uniformly to $g^{\prime}$. We deduce that $\left(\begin{array}{c}u \\ z\end{array}\right) \in C^{1}\left([0, T] ;\left(L^{2}(\mathbb{T})\right)^{2}\right)$.

Taking into account the form of $\left(\begin{array}{l}u \\ z\end{array}\right)$ given by (36), it follows that (4) holds.

With similar arguments as in the proof of Theorem 3.1, it follows that $\left(\begin{array}{l}u \\ z\end{array}\right)$ is unique solution of (4), and the proof is complete.

\section{References}

[1] B. D. Coleman and M. E. Gurtin, Equipresence and constitutive equations for rigid heat conductors, Z. Angew. Math. Phys. 18, 199-208 (1967).

[2] P. L. Davis, On the linear theory of heat conduction for materials with memory, SIAM J. Math. Anal. 9, 49-53 (1978).

[3] M. E. Gurtin and A. C. Pipkin, A general theory of heat conduction with finite wave speeds, Arch. Rational Mech. Anal. 31, 113-126 (1968).

[4] A. W. Knapp, Basic real analysis (Birkhäuser, Boston, 2005) 680 pp.

[5] J. W. Nunziato, On heat conduction in materials with memory, Quart. Appl. Math. 29, 187-204 (1971). 